\title{
Electron Microscopy and Spectroscopy of Au-coated Carbon Nanotubes for Electron Field Emission Applications
}

\author{
Joshua M. Green, Jun Jiao*
}

Department of Physics, Portland State University P.O. Box 751, Portland, Oregon 97207

*Corresponding author. E-mail: jiaoj@pdx.edu

Since their discovery in 1991 [1], carbon nanotubes (CNTs) have been investigated as electron field-emission sources for use in devices ranging from flat-panel displays to electron microscopes. Lately there has been much interest in CNT/metal composites, especially CNTs decorated with metal nanoparticles. Although the attachment of nanoparticles to CNTs has been studied extensively, most methods involve a tedious assembly process in chemical solution, using catalyst materials or functionalization. In this study, we combined CNTs and Au directly together to form $\mathrm{Au}$-coated CNTs and studied their electron field emission properties.

CNTs were grown using Fe as a catalyst. Silicon substrates coated with Fe catalyst were placed in a ceramic boat in a quartz tube, which was placed in a horizontal tube furnace. The samples were heated to $700^{\circ} \mathrm{C}$ at 75 Torr of an admixture of $\mathrm{H}_{2}(385 \mathrm{sccm})$ and $\mathrm{C}_{2} \mathrm{H}_{2}(25 \mathrm{sccm})$ and kept at that temperature for $30 \mathrm{~min}$. When the reactor reached room temperature, the samples were removed from the CVD furnace and a thin film of a nominally $50 \mathrm{~nm}$ thickness of Au was deposited using a sputtering process. The samples were subsequently annealed in Ar at $750^{\circ} \mathrm{C}$ for 60 seconds.

Figure 1a shows a scanning electron microscope (SEM) image of the as-grown CNTs, while figure $1 \mathrm{~b}$ reveals the CNT carpet after deposition and annealing of Au. Note the Au seems to agglomerate in particles on the surface of the CNTs. Figure 1c shows a TEM image of a Au particle attached to a CNT, which confirms the Au particles are roughly the same diameter as the bare CNTs. An energy-dispersive X-ray spectrometer (EDX) was employed to determine the chemical composition of the samples, shown in figure $1 \mathrm{~d}$.

Electron field emission measurements were performed in an ultrahigh vacuum (UHV) system. A Keithley 248 high voltage supply provided a voltage increasing with a sweep step of $50 \mathrm{~V}$. The field emission current was monitored by a Keithley 2400 sourcemeter. The field emission properties of both as-grown CNTs and Au-coated CNTS were measured with fixed separation distances of 100 $\mu \mathrm{m}$ between the anode and the cathode, where the emitting area of each sample was approximately 1 $\mathrm{mm}^{2}$. The curves of field emission current density $(J)$ versus electric field $(E)$ are shown in Figure 2. The threshold current density $\left(1 \mathrm{~mA} / \mathrm{cm}^{2}\right)$ for the as-grown CNTs was achieved at $8.2 \mathrm{~V} / \mu \mathrm{m}$, while the threshold field for the Au-coated CNTs was $6.0 \mathrm{~V} / \mu \mathrm{m}$. It is clear from figure 2 that for a given electric field, the Au-coated CNTs have a higher current density; the highest current density for the Au-coated CNTs is $1550 \mathrm{~mA} / \mathrm{cm}^{2}$ at an applied field of $25 \mathrm{~V} / \mu \mathrm{m}$, while at that same applied field, the current density of as-grown CNTs is only $16.9 \mathrm{~mA} / \mathrm{cm}^{2}$. Previous studies have shown an improvement in field emission properties from a change in geometry [2]. Since the Au particles are roughly the same diameter as the bare CNTs, it is conjectured that the Au particles act as additional emission sites, thus increasing the emission density. Our study, therefore, demonstrates a simple way to increase the effectiveness of field emitters by changing their shape.

Financial support for this research was provided in part by the NSF under awards No. ECS-0348277 and ECS-0722660.

References

[1] S. Iijima, 1991, Nature, 354, 56

[2] M. Sveningsson, R.E. Morjan, O.A. Nerushev, E.B. Campbell, D. Malsch, and J.A. Schaefer, 2004, Appl. Phys. Lett., 85, 4487. 

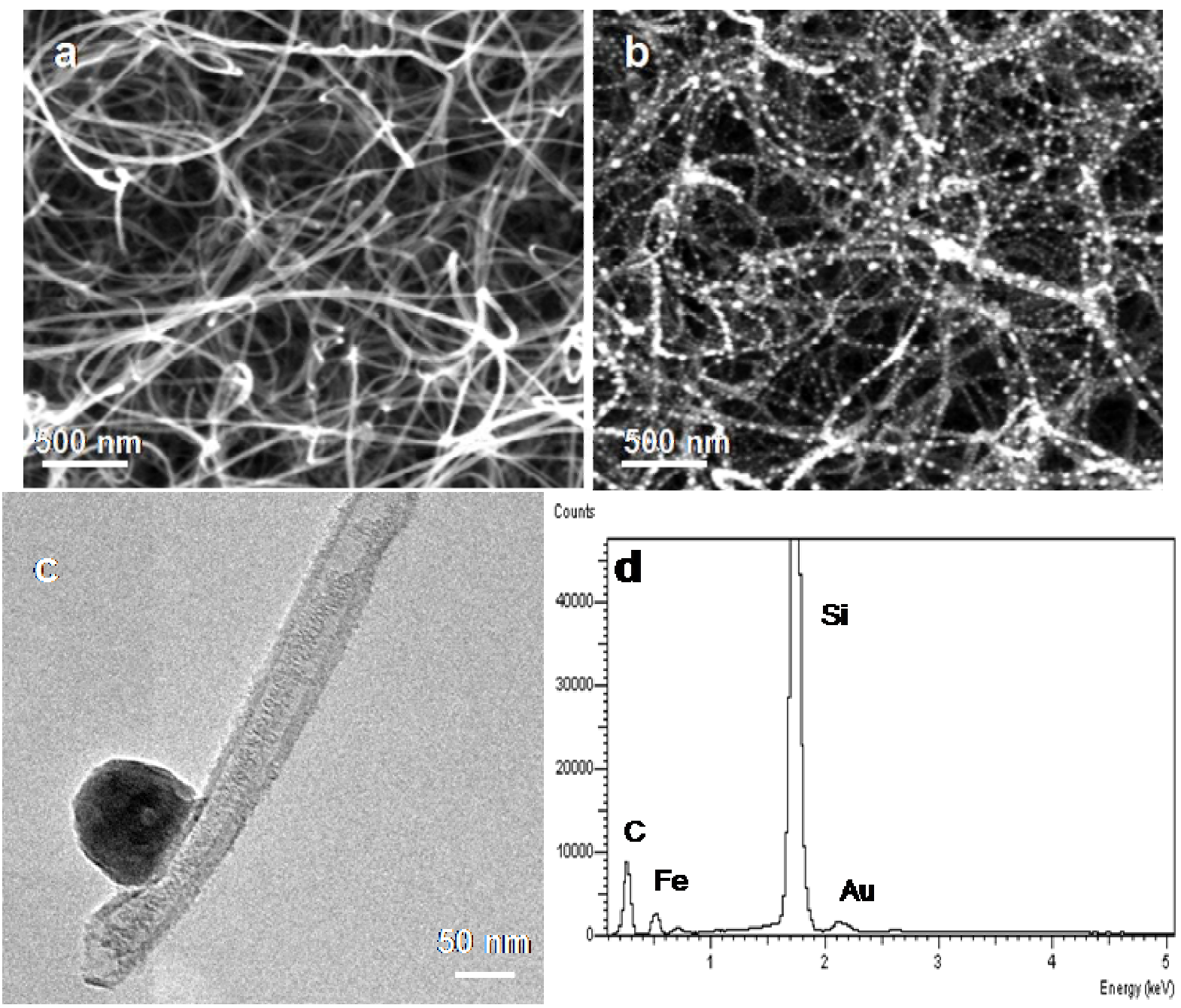

Figure 1 SEM images of (a) as-synthesized CNTs, (b) carbon nanotubes coated with a thin layer of Au and annealed, (c) TEM image of a CNT with a Au particle attached and (d) EDX spectra of Aucoated CNTs. The Si signal is from the substrate and the Fe signal is from the catalyst.

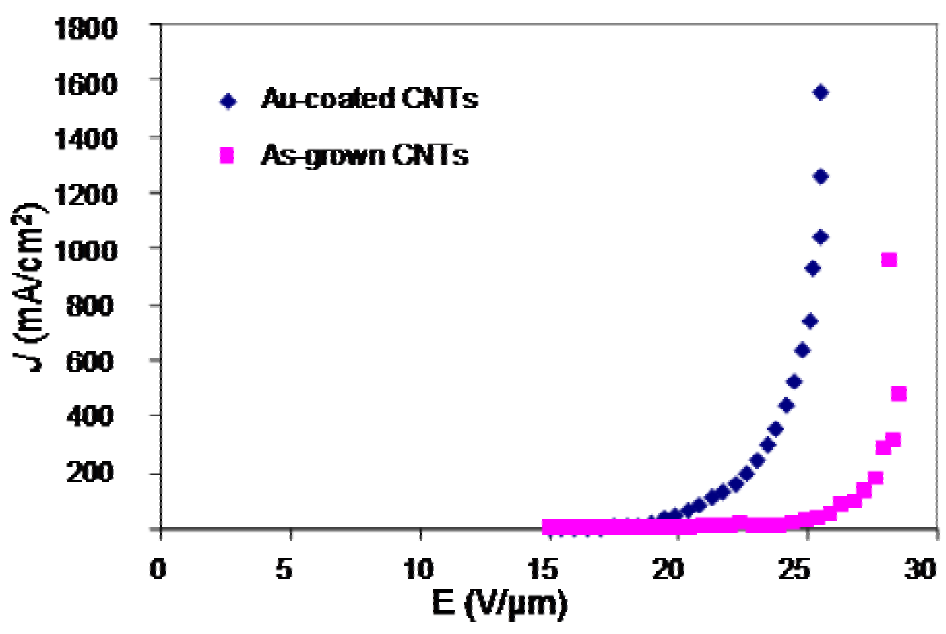

Figure $2 J-E$ curves of as-grown CNTs and Au-coated CNTs 